\title{
Prevalencia de neuromitos en docentes de la Universidad de Cienfuegos
}

\author{
Neuromyth prevalence in teachers at the University of Cienfuegos
}

\section{Prevalência de neuromito em professores da Universidade de Cienfuegos}

\author{
Elena Hatty Jiménez Pérez ${ }^{1}$, ORCID 0000-0003-3257-3164 \\ Oscar Ovidio Calzadilla-Pérez ${ }^{2}$, ORCID 0000-0003-4322-3098 \\ ${ }^{1}$ Universidad Autónoma de Baja California. México \\ ${ }^{2}$ Facultad de Educación, Universidad Católica de Temuco. Chile
}

\begin{abstract}
Resumen: La aplicación prematura del conocimiento neurocientífico genera creencias erróneas o neuromitos en docentes. De ahí la ejecución de un estudio descriptivo dirigido a identificar la prevalencia de neuromitos en 40 docentes universitarios de la Facultad Ciencias de la Educación de la Universidad de Cienfuegos (Cuba) y los predictores que pueden influir en las falsas creencias acerca del cerebro. A tales efectos se contextualizó un cuestionario diseñado para evaluar neuromitos y predictores. Se ejecutó un análisis de las variables: 1) características de los participantes, 2) neuromitos, y 3) predictores; para lo que se aplicó la regresión lineal. La evaluación de la hipótesis de estudio se realizó a través de la prueba no paramétrica Chi Cuadrado de Bondad de Ajuste. El resultado más significativo fue la identificación de predictores sobre el predominio de neuromitos en los docentes para su posterior tratamiento en la formación inicial y continua.
\end{abstract}

Palabras clave: neurociencias; educación superior; neuromitos

Abstract: The premature application of neuroscientific knowledge generates erroneous beliefs or neuromyths in professors. Hence the execution of a descriptive study aimed at identifying the prevalence of neuromyths in 40 university professors from the Faculty of Education Sciences of the University of Cienfuegos (Cuba) and the predictors that may influence false beliefs about the brain. For this purpose, a questionnaire designed to evaluate neuromyths and predictors was contextualized. An analysis of the variables was executed: 1) participants' characteristics, 2) neuromyths, and 3) predictors; for which linear regression was applied. The evaluation of the study hypothesis was carried out through the nonparametric Chi-Square Goodness-of-Fit Tests. The most significant result was the identification of predictors on the predominance of neuromyths in professors for their subsequent treatment in initial and continuous training.

Keywords: neurosciences; higher education; neuromitos 
Resumo: A aplicação prematura de conhecimentos neurocientíficos gera crenças errôneas ou neuromitos em docentes. A partir daí a realização de um estudo descritivo com o objetivo de identificar a prevalência de neuromitos em 40 professores universitários da Faculdade de Ciências da Educação da Universidade de Cienfuegos (Cuba) e os preditores que podem influenciar falsas crenças sobre o cérebro. Para tanto, foi contextualizado um questionário elaborado para avaliar neuromitos e preditores. Foram realizadas análises das variáveis: 1) características dos participantes, 2) neuromitos e 3) preditores; para as quais a regressão linear foi aplicada. A avaliação da hipótese do estudo foi realizada por meio do teste não paramétrico Chi Square Goodness of Fit. O resultado mais significativo foi a identificação de preditores sobre a predominância de neuromitos em professores para seu posterior tratamento na formação inicial e continuada.

Palavras-chave: neurociências; educação superior; neuromitos

Recibido: 24/05/2020

Aceptado: 23/11/2020

\section{Cómo citar:}

Jiménez Pérez, E. H., \& Calzadilla-Pérez, O. O. (2021). Prevalencia de neuromitos en docentes de la Universidad de Cienfuegos. Ciencias Psicológicas, 15(1), e-2358. doi: https://doi.org/10.22235/cp.v15i1.2358

Correspondencia: Elena Hatty Jiménez Pérez, Universidad Autónoma de Baja California, Av. Río Santa María, 2081, Paseos del Sol, CP: 21399, Mexicali, Baja California, México. Email: hattycfg@gmail.com Oscar Ovidio Calzadilla Pérez, Universidad Católica de Temuco, Avenida Rudecindo Ortega 02950, Temuco, La Araucanía, Chile, CP: 478000. Email: calzadilla1984@uct.cl

El interés de investigadores y docentes por vincular las neurociencias y las ciencias de la educación a partir del establecimiento de lo que Bruer denomina puentes, constituye una problemática teórica y de orden práctico en ascenso continuo (Bruer, 1997, 2016). Los criterios resultantes de la indagación científica al respecto muestran un punto para la generación de investigaciones básicas y aplicadas, entre las que existe un nodo común: el valor agregado del conocimiento neurocientífico para la práctica educativa como vía para promover un desarrollo humano y social sostenible (Calzadilla-Pérez, 2017; Castorina, 2016; Fernández, 2017; Román, 2013; Zuluaga, 2018).

En este contexto de encuentros entre conocimientos educativos y neurocientíficos los neuromitos devienen en teorías relativamente falsas, sin base científica suficiente, y que por su recurrencia se instalan en las representaciones sociales de los sujetos (Barraza \& Leiva, 2018; Ferreira, 2018; Ferreira \& Gómez, 2019; Nancekivell, Shah \& Gelman, 2020; Román, 2013). Estos apuntan hacia métodos de enseñanza, estilos y actividades novedosas que intentan desarrollar aprendizajes basados en el funcionamiento del cerebro y, en el peor de los casos, sin una sistematización previa de criterios científicos y su transferencia crítica. Varios de los neuromitos que persisten en la actualidad han surgido de la divulgación de resultados de laboratorios, lo que dista de los ambientes del aula en la que el docente educa, instruye y enseña en condiciones naturales.

En este sentido resulta pertinente referir la maduración plena de disciplinas científicas emergentes como son la Neuroeducación, la Neuropedagogía y la Neurodidáctica, como expresión del encargo común de investigadores y docentes por crear nuevos puentes de 
integración de conocimientos con fundamentación científica que desmientan las falsas creencias. Si bien estas disciplinas no cuentan con consenso absoluto en su reconocimiento, los nuevos conocimientos que se generan en proyectos de investigación, el aumento de programas de maestría y doctorado, y la cantidad de publicaciones ascendente, muestran su pertinencia (Battro, Fischer \& Pierre, 2008; Blakemore \& Frith, 2007; Codina, 2014; Geake, 2005; Goswami, 2004, 2006; Howard-Jones, 2010; Mora, 2013).

En esta línea de pensamiento se encontraron estudios precedentes como los de Beiras (1998), quien afirmó que después de la puesta en práctica de módulos de Neurociencias y Neurodidáctica los estudiantes tienden a mostrar una actitud favorable para aceptar la información neurocientífica, al considerar su carácter interdisciplinar. Román (2013) concluyó la existencia de docentes que todavía piensan en las Neurociencias como parte exclusiva de la medicia. Por su parte, López (2017) defiende que los conocimientos neurocientíficos tienen una influencia significativa en el componente cognitivo, afectivo y conativo de las actitudes de los docentes en formación. Estos criterios coinciden en algunos puntos de análisis de Zabalza (2018), quien al referirse a las aportaciones de las Neurociencias a la Educación Infantil, plantea

(...) la necesidad de incorporar esa temática a los Planes de Estudio de los futuros educadores y educadoras de niños pequeños. [...] Desgraciadamente poco a poco se han ido pedagogizando en exceso las carreras de preparación de docentes y educadores y perdiendo esos otros aportes esenciales para poder entender el desarrollo infantil (2018, p. 83).

De hecho, la investigación aplicada pondera como una de las cusas respecto de la proliferación de los neuromitos el débil nivel de tratamiento del conocimiento de las neurociencias en las mallas curriculares para la formación de docentes. Pese a lo planteado en las dos últimas décadas persiste el interés de expertos y docentes por su integración en la formación del profesional de la educación. Esta afirmación es palpable en los resultados de las investigaciones desarrolladas por Bacigalupe y Mancini (2014), Calzadilla-Pérez (2015, 2017), Calzadilla-Pérez et al. (2018), Carvalhoa y Villas (2018), Horvath y Donoghue (2016), López (2017), Luque y García (2017), Martín (2012), Melo (1998), Román (2013, 2018), Tapia (2013), y Resende y Colombo (2018).

Contradictoriamente, los docentes tienen entre sus retos revertir la prevalencia de los neuromitos mediante la transferencia y validación pedagógica del conocimiento neurocientífico, con el propósito de transformar la práctica en sus aulas desde el dominio de la teoría. Este propósito aumenta su envergadura cuando se trata de formación de maestros en la educación superior.

Conforme al hecho de que el conocimiento neurocientífico es parte del derecho social a la información, la investigación al respecto connota que en la actualidad los profesionales de la educación muestran una actitud flexible por la mejora gradual de la enseñanza sustentada en este conocimiento; sin embargo, la multiplicación de neuromitos como práctica, limita la concreción de este desafío (Ansari, De Smedt \& Grabner, 2012). Esta preocupación ha generado investigaciones sobre la prevalencia de neuromitos en estudiantes y profesores de varios países, las que se concentran en Europa y Norteamérica, con un número menor en Latinoamérica (Lipina, 2016).

De hecho, las indagaciones sobre prevalencia de conocimientos erróneos o neuromitos en docentes de distintos países demuestran la pertinencia de su estudio, lo cual se concibe para los autores como preámbulo para el perfeccionamiento de programa\&s de formación. Al respecto, es ilustrativo mencionar los trabajos realizados en países como Reino Unido y Holanda (Dekker, Lee, Howard-Jones \& Jolles, 2012), Portugal (Rato, Abreu, \& Castro-Caldas, 2013), Turquía (Karakus, Howard-Jones \& Jay, 2015), China (Pei, Howard-Jones, Zhang \& Liu, 2014), Suiza (Tardif, Doudin \& Meylan, 2015), España (Fuentes \& Risso, 2015) y Ecuador (Falquez \& Ocampo, 2018). 
En la búsqueda de causas que favorecen la presencia de neuromitos en docentes universitarios cubanos, se ponderan los criterios resultantes de dos estudios previos. En el primero se afirma que "la integración del conocimiento neurocientífico en la educación se ha caracterizado por la insuficiente argumentación pedagógica y didáctica, y relativa descontextualización a las particularidades de las etapas del desarrollo por las que transita el sujeto en su ontogénesis" (Calzadilla, 2017, p. 1). En el segundo estudio (Calzadilla-Pérez et al., 2018) se concluyó que, si bien la integración del conocimiento neurocientífico no tiene un tratamiento suficientemente explícito en los programas para la formación de maestros, su dominio es contenido de la formación pedagógica que amerita del tratamiento didáctico en programas de disciplinas y asignaturas. Sin embargo, entre las causas que han limitado su tratamiento más explícito e intencional se valoran a las siguientes:

- Limitaciones en el acceso a los resultados de investigaciones extranjeras y cubanas que aportan conocimientos neurocientíficos a la educación, lo que limitó la visión pedagógica de su valor agregado en el proceso educativo y la formación de una cultura interdisciplinaria en los profesores universitarios que permitiera fundamentar a la educación, también desde sus bases biológicas.

- Fragmentación de conocimientos neurocientíficos en el diseño curricular y débil búsqueda de nodos y relaciones interdisciplinarias, desde el trabajo metodológico en los niveles organizativos de la carrera, lo que limitó su integración y tratamiento gradual en la solución de problemas profesionales y la asimilación de contenidos para la satisfacción de las funciones y tareas de su rol, desde las disciplinas y años académicos.

Lo planteado explica la necesidad de ofrecer tratamiento en el diseño curricular de las carreras, en programas de superación profesional y de formación académica para docentes a los fundamentos que singularizan la integración del conocimiento neurocientífico como condición sine qua non para comprender, argumentar y demostrar el carácter espontáneo y empírico de los neuromitos que prevalecen en docentes universitarios.

En consecuencia, el objetivo de este trabajo radica en: determinar la prevalencia de neuromitos en docentes universitarios de la Facultad de Educación de la Universidad de Cienfuegos, por cuanto el dominio de este conocimiento sienta las bases para la ejecución de programas de superación, de futuras líneas de investigación y el enriquecimiento de la gestión didáctica en los colectivos docentes a nivel de asignaturas, disciplinas académicas y carreras.

\section{Materiales y Métodos}

Se realizó un estudio de tipo descriptivo, transversal (Hernández, Fernández, \& Baptista, 2014). El período del estudio es realizado entre los meses de septiembre y diciembre de 2018 en la Facultad de Ciencias de la Educación de la Universidad de Cienfuegos.

\section{Población y Muestra}

La muestra es de tipo probabilística, y se procedió a partir del muestreo aleatorio simple. Esta se conformó por 40 docentes que estuvieron presentes el día de la aplicación del instrumento en los departamentos adscritos a la Facultad de Educación. De esta forma queda representada con un $82.5 \%(n=33)$ Licenciados en Educación y $17.5 \%(n=7)$ Licenciados en carreras de Ciencias Técnicas. El rango de edad de los encuestados es de 23 y 67 años $(M=$ $46.20, D E=14.21)$ y de experiencia laboral en educación es desde 3 meses hasta 47 años $(M=$ 2.62, $D E=14.97)$. De estos, 5 fueron de sexo masculino, y 35 de sexo femenino.

En la caracterización de los participantes se corroboró que la mayor cantidad ostentan el grado científico de Doctor en Ciencias y la categoría docente principal de Profesor Auxiliar. El $82.5 \%$ se tituló en su formación de pregrado en carreras de ciencias de la educación, y solo el 
$17.5 \%$ en carreras de ciencias técnicas, estos últimos pertenecen a la Facultad de Educación debido a que alcanzan en su formación de postgraduados titulaciones académicas en educación; además, todos están vinculados a la formación de docentes. Es significativa la composición categorial de los participantes en tanto el $72.5 \%$ se formaron como Máster o Doctor en Ciencias en una disciplina específica, y el 52.5\% ostentan las categorías docentes principales de Profesor Auxiliar o Profesor Titular.

\section{Instrumento}

Se realizó una adaptación al instrumento diseñado por Dekker et al. (2012) en su versión en idioma español, quedando conformado por 25 ítems, 14 correspondientes al estudio del conocimiento sobre neuromitos y 11 al dominio de aspectos generales acerca del cerebro. Conforme al instrumento original, los ítems tienen enfoque de preguntas semicerradas con las opciones de respuesta verdadero (V), falso (F) o no sé (NS). Además, se añadieron para el estudio preguntas generales tomadas de la encuesta elaborada por Falquez y Ocampo (2018) que consisten en ubicar en una escala Likert del 1 (equivalente a muy bajo) al 7 (equivalente a muy alto), el nivel de autovaloración de su desempeño como docente universitario, el nivel de interés por la Neuroeducación, el conocimiento sobre esta y el nivel de seguridad en responder.

\section{Procedimiento}

En un primer momento se les notificó a los participantes por vía email sobre su disponibilidad de horario para la contestación de un instrumento que formaba parte de una investigación en curso y la necesidad de firmar un consentimiento informado donde otorgan su permiso a la producción parcial y total de sus respuestas, manteniendo el anonimato. Luego se procedió a la aplicación de las encuestas en formato impreso, donde los docentes daban respuesta con su puño y letra. Se realizaron en una semana en horario laboral y los investigadores acompañaron en todo momento a los docentes participantes. Sin embargo, no fue posible que la población objeto de estudio participara en su totalidad, por cuestiones de tiempo, ritmos de trabajo y acceso al correo donde se dió aviso previo. Asimismo, la investigación forma parte del diagnóstico educativo de una tesis de maestría y ello facilitó la aprobación y aval del comité científico de la Maestría en Educación de la Universidad de Cienfuegos.

\section{Análisis de datos}

Los datos recolectados se analizan a través del procesador estadístico Statistical Package for the Social Sciences (SPSS) en su versión 22.0 para Windows. Teniendo en consideración que la hipótesis de trabajo se ciñe a la determinación de la prevalencia significativa de neuromitos en docentes universitarios de la Facultad de Ciencias de la Educación de la Universidad de Cienfuego, las variables estudiadas son: 1) neuromitos, 2) predictores de neuromitos y 3) conocimientos generales acerca de la Neurociencia. La primera se analiza mediante la estadística inferencial con la prueba no paramétrica Chi Cuadrado de bondad de ajuste con el propósito de evaluar la hipótesis. La segunda, es analizada a través de la regresión lineal donde la variable dependiente fueron los neuromitos que prevalecieron en los participantes y la tercera mediante la estadística descriptiva.

\section{Resultados}

Los resultados muestran la descripción de las variables: 1) prevalencia de neuromitos, $y$, 2) conocimientos generales acerca de la Neurociencia y predictores de los neuromitos.

Tras la aplicación del instrumento se constató que el mayor número de participantes (67.5\%) declaran no haber recibido capacitación en relación con las neurociencias aplicadas a la educación, lo que evidencia un sesgo respecto al tratamiento de núcleos rectores del actual Plan 
de Estudio "E" en el que "el conocimiento anatomofisiológico se percibe en el programa de la disciplina Formación Pedagógica General un núcleo integrador" (MES, 2016). Sin embargo, más de la mitad de la muestra $(60 \%)$ mencionó tener un alto nivel de interés por la Neuroeducación, aunque menos de la mitad (35\%) reconoció no tener conocimientos acerca de esta.

El $97.5 \%$ declaró que practicaban la lectura de publicaciones populares de ciencia y un $82.5 \%$ la lectura de revistas científicas, en las que se visibiliza conocimiento sobre Neuroeducación. Por otra parte, la mitad de los participantes valoraron como "muy alto" su desempeño como docente. Asimismo, seis de cada diez participantes valoraron de "alto" y "muy alto" el nivel de seguridad en responder las preguntas del cuestionario. Esto demuestra la tendencia de la muestra hacia valores altos en la escala del instrumento.

Los neuromitos más prevalentes en los docentes fueron los siguientes:

- N 7. Los alumnos aprenden mejor cuando reciben la información en su estilo de aprendizaje preferido (auditivo, visual y kinestésico), en un $90 \%$ de los participantes.

- N 8. Las sesiones cortas de ejercicios de coordinación pueden mejorar la integración de la función cerebral de los hemisferios, con una prevalencia en el 70 $\%$ de la muestra.

Otros de los neuromitos considerados medianamente prevalentes son:

- N 10. Los entornos que son ricos en estímulos mejoran el desarrollo del cerebro de los niños y niñas de la primera infancia, con un $97.5 \%$.

- N 12. Los ejercicios físicos que promueven la coordinación de las habilidades perceptivo-motoras pueden mejorar las destrezas en lecto-escritura, en un $87.5 \%$ de los docentes.

- N 14. Para que el aprendizaje sea aún más efectivo se deben estimular las habilidades del HD y el HI $(67.5 \%)$.

En la tabla 1 se muestran los porcentajes que presentan los neuromitos, respecto a la pregunta en la que los docentes debían responder con las respuestas de verdadero (V), falso (F) o no sé (NS). Obsérvese el predominio de los neuromitos 7, 10 y 12, así como el nivel de información que los docentes no dominan para fundamentar desde la ciencia los neuromitos 7 , 10 y 12 , fundamentalmente.

Tabla 1

Prevalencia de neuromitos

Neuromitos

V

$\mathrm{F}$

N 7. Los alumnos aprenden mejor cuando reciben la información en su estilo de aprendizaje preferido (auditivo, visual, kinestésico)

$90 \% \quad 7.5 \%$

$2.5 \%$

N 8. Las sesiones cortas de ejercicios de coordinación pueden mejorar la integración de la función cerebral de los hemisferios

$70 \%$

$30 \%$

$\mathrm{N}$ 10. Los entornos que son ricos en estímulos mejoran el desarrollo del cerebro de los niños de la primera infancia

$97.5 \%$

$2.5 \%$

$\mathrm{N}$ 12. Los ejercicios físicos que promueven la coordinación de las habilidades perceptivo-motoras pueden mejorar las destrezas en lecto- $87.5 \%$ $12.5 \%$ escritura

N 14. El aprendizaje más efectivo tiene como condición estimular las habilidades del hemisferio derecho y el hemisferio izquierdo

$65.5 \% \quad 5 \%$ $27.5 \%$ 
Finalmente, los resultados de la prueba no paramétrica Chi Cuadrado de bondad de ajuste, como la $\mathrm{X}^{2}$ calculado $=57.95 ; 27.59 ; 55.51 ; 42.72 ; 22.52>\mathrm{X}^{2}$ Teórico $=5.9914, p>.05 \mathrm{se}$ puede rechazar la hipótesis nula y afirmarse, con un nivel de significatividad de $95 \%$, que es significativa la prevalencia de cinco neuromitos en los docentes universitarios participantes en este estudio.

En relación con los conocimientos sobre Neurociencias en los que los docentes mostraron mayor desconocimiento se encuentran los siguientes (ver también Tabla 2):

- el logro académico puede verse afectado al saltarse el desayuno;

- los ritmos circadianos (el reloj biológico) cambian durante la adolescencia, causando que los alumnos se sientan cansados durante las primeras clases del día;

- se ha demostrado científicamente que los suplementos de ácidos grasos (omega 3 y 6) tienen un efecto positivo en el rendimiento académico; y,

- los niños se muestran inatentos después de consumir bebidas azucaradas o golosinas.

Tabla 2

Conocimientos generales sobre neurociencias

\begin{tabular}{lccc}
\hline \multicolumn{1}{c}{ Ítems } & $\mathrm{V}$ & $\mathrm{F}$ & $\mathrm{NS}$ \\
\hline El logro académico puede verse afectado al saltarse el desayuno & $47.5 \%$ & $32.5 \%$ & $20 \%$ \\
\hline $\begin{array}{l}\text { Los ritmos circadianos (el reloj biológico) cambian durante la } \\
\text { adolescencia, causando que los alumnos se sientan cansados durante las } \\
\text { primeras clases del día }\end{array}$ & $42.5 \%$ & $15 \%$ & $42.5 \%$ \\
\hline
\end{tabular}

Se ha demostrado científicamente que los suplementos de ácidos grasos (omega 3 y 6) tienen un efecto positivo en el rendimiento académico

$30 \% \quad 10 \% \quad 60 \%$

\begin{tabular}{llllllll}
\hline Los niños se muestran inatentos después de consumir bebidas & $22.5 \%$ & $30 \%$ & $47.5 \%$
\end{tabular}

Por otra parte, tal como se muestra en la Tabla 3 el análisis de regresión lineal reveló que el nivel de interés por la Neuroeducación es un predictor significativo $(\beta=0.03 ; p<.05)$ de los cinco neuromitos que prevalecieron en el presente estudio.

Tabla 3

Predictores de los neuromitos

\begin{tabular}{l|c|c|c|c}
\hline Predictores & $\beta$ & $\mathrm{DE}$ & $\mathrm{T}$ & Sig. \\
\hline Interés por la neurociencia educacional & 0.332 & 0.153 & 2.166 & $.038^{*}$ \\
\hline Edad & 0.023 & 0.012 & 1.048 & .063 \\
\hline Años de experiencia & 0.007 & 0.004 & 1.761 & .086 \\
\hline Capacitación en neurociencias & 0.260 & 0.143 & 1.811 & .079 \\
\hline Conocimiento sobre neurociencia educacional & 0.405 & 0.224 & 1.803 & .081 \\
\hline
\end{tabular}

Asimismo, no son recurrentes los neuromitos que hacen referencia a las diferencias en el aprendizaje de los niños después de consumir bebidas o alimentos azucarados, ya que solo un $22.5 \%$ afirma que es verdadero. Respecto al efecto positivo en el logro académico de los suplementos de ácidos grasos (omega-3 u omega-6) afirman como verdadero el $30 \%$. Por lo que, 
se puede afirmar que en los docentes universitarios cubanos encuestados solo prevalecen cinco de los siete neuromitos más predominantes según Dekker et al. (2012).

\section{Discusión}

En relación con los predictores, no se muestra similitud con las investigaciones precedentes ya que el predictor en este caso es el interés por la Neuroeducación. Esto significa que a mayor interés que tienen los docentes por la Neuroeducación, mayor es la creencia en neuromitos.

Si bien la contradicción típica entre lo desconocido y los nuevos conocimientos neurocientíficos en un marco temporal específico, se puede afirmar es uno de los factores epistémicos que condicionan el surgimiento de neuromitos, otra de las causas radica en el desmedido y subjetivo interés por aplicar de forma acrítica los avances en neurociencias en la educación.

Asimismo, la explosión de información sobre el cerebro ha causado que existan falsas interpretaciones sobre sus aportes y la invisibilización de las aplicaciones de la Neuroeducación (Goswami, 2006). Por otra parte, la brecha que existe entre investigadores, neurocientíficos y docentes ha facilitado la rápida proliferación en los centros educativos de los llamados neuromitos, o conceptos erróneos en torno al cerebro (Howard-Jones, 2014). En este marco, se aseguran consecuencias que demuestran la existencia de programas educativos, supuestamente, basados en la neurociencia que carecen de evidencia científica que demuestren su eficacia.

Cada una de estas creencias distorsionadas de la realidad, además de proliferar aprendizajes especuladores, sitúan a los docentes en muestras etiquetadas debido al desconocimiento neurocientífico. Esto propicia la creación de actividades, programas y ejercicios sin fundamentos científicos sólidos para la educación, principalmente en la primera infancia (Gleichgerrcht, Luttges, Salvarezza \& Campos, 2015).

Este estudio tiene entre sus valores agregados constituir una evidencia científica para generar investigaciones que ofrezcan argumentos convincentes para mitigar las falsas teorías acerca del cerebro. En este contexto de análisis se realiza un análisis comparado del promedio de prevalencia de neuromitos por países según los datos ofrecidos por Falquez y Ocampo 82018) respecto a la obra de Dekker et al. (2012), Ferrero y otros (Fuentes \& Risso, 2015) y Gleichgerrcht et al. (2015) tal como se muestra en la figura 1.

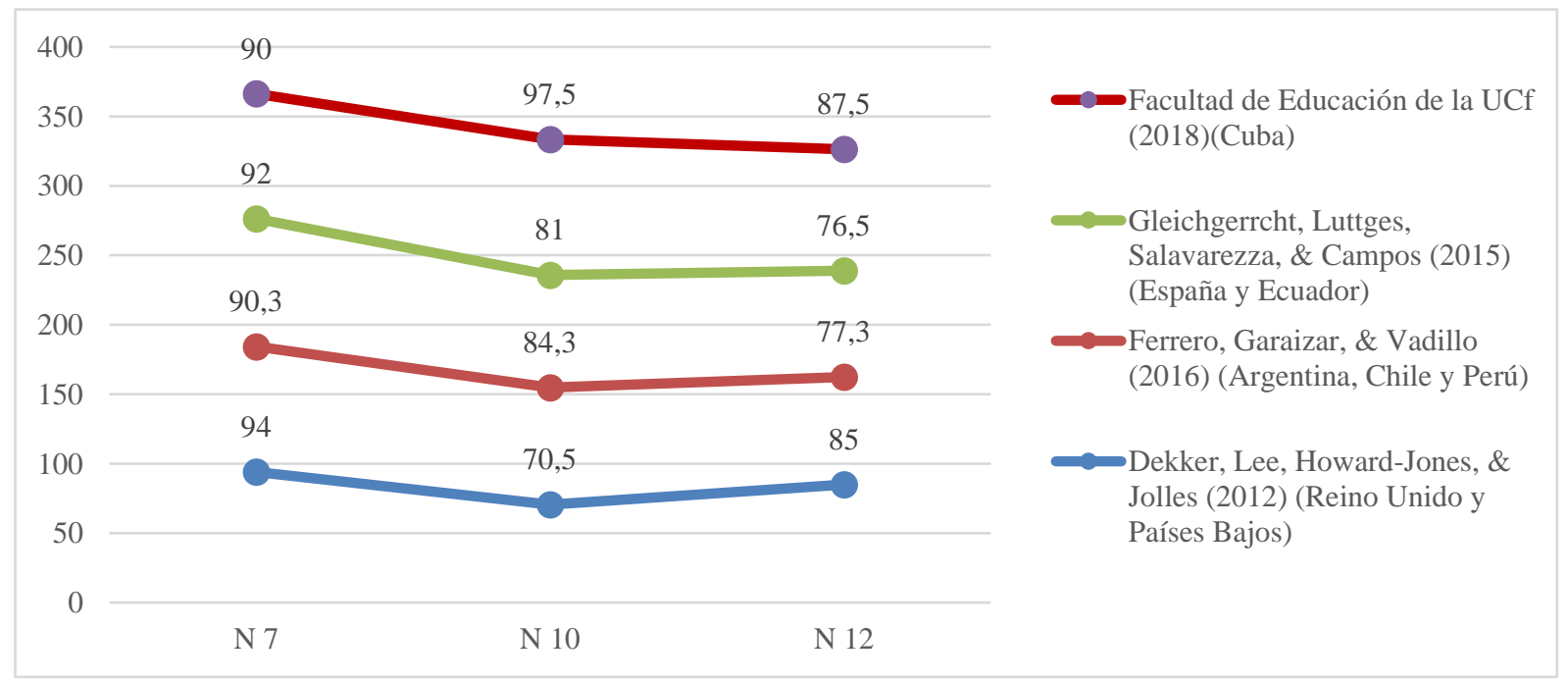

Figura 1. Resultados de estudios de referencia sobre prevalencia de neuromitos 
En efecto los datos muestran el N 7 como el de mayor prevalencia de los estudios de referencia en la Figura 1, con un índice porcentual poco significativo en cuanto la diferencia entre estos. El N 10 y el N 12 son los otros dos con mayor por ciento de prevalencia en todos los casos, y los índices porcentuales hacia su interior son mayores. En general, el resultado de esta investigación coincide con el promedio de prevalencia de los países en los que realizan los estudios de referencia.

Sin embargo, no es coincidente que respecto al por ciento el estudio cubano muestre cifras mayores, y entre los factores que lo condicionan, además de los referenciados con anterioridad en este mismo trabajo de Calzadilla-Pérez et al. (2018), se encuentra el hecho de que la Neuroeducación y sus ramas vertebrales afines como la Neuropedagogía y la Neurodidáctica no han logrado afianzarse como líneas de trabajo metodológico y de investigación sólidas. De ahí que, la sistematización pedagógica del conocimiento neurocientífico en los currículos para la formación de docentes no constituye una arista direccionada y prioritaria de las comisiones nacionales de carrera.

\section{Conclusiones}

Los resultados alcanzados permitieron determinar la prevalencia de neuromitos en docentes universitarios de la Facultad de Educación de la Universidad de Cienfuegos. Los conocimientos de mayor dificultad y el predictor de los neuromitos que prevalecen en los participantes. Aunque, en este caso el número de neuromitos fue menor que los encontrados en docentes de otros países, las dificultades en los conocimientos tienden a ser mayores. Asimismo, el predictor interés por la Neuroeducación revela docentes motivados por esta disciplina. Los estudios revisados en el presente trabajo permitieron hacer una relación entre los resultados aportados para una muestra determinada de profesionales de la educación de otros países y los profesionales cubanos pertenecientes a la Universidad de Cienfuegos. Ello corroboró que los docentes universitarios cubanos encuestados no tienen neuromitos que en otras muestras aseguran prevalencia, lo cual puede encontrar respuesta en la integración de las neurociencias en congresos internacionales y nacionales que tienen lugar en la universidad en el último año donde se presentan temas que abordan esta disciplina y sus aportes a las ciencias de la educación.

Sin embargo, se concluye que la prevalencia de neuromitos en los docentes del estudio tiene su causa fundamental en la débil integración y tratamiento de conocimientos neurocientíficos en las mallas curriculares. Esto se refleja en la formación inicial y en la oferta de educación continua. En este contexto la carencia de proyectos de investigación que manejen como variable de estudio a la evaluación de los currículos de formación docente y genere la actualización científica de estos con contenidos de las neurociencias deviene entre las causas, tal como se expuso en las investigaciones de Calzadilla-Pérez $(2017,2018)$.

Los resultados expuestos tienen entre sus valores agregados ofrecer información para generar futuras investigaciones para la mejora curricular desde las funciones de las comisiones nacionales de carrera y los claustros universitarios.

\section{Referencias}

Ansari, D., De Smedt, B. \& Grabner, R. (2012). Neuroeducation-A Critical Overview of An Emerging Field. Neuroethics, 5(2), 105-117. doi: 10.1007/s12152-011-9119-3

Bacigalupe, M. \& Mancini, V.A. (2014). Contribuciones para la construcción de un enfoque de las neurociencias de y con la educación en la formación universitaria de pregrado en 
ciencias de la educación. Profesorado: Revista de Currículum y Formación del Profesorado, 18(3), 431-440.

Barraza, P., \& Leiva, I. (2018). Neuromitos en educación: Prevalencia en docentes chilenos y el rol de los medios de difusión. Paideia, (63), 17-40.

Battro, A., Fischer, K. \& Pierre, L. (Eds.) (2008). The educated brain: Essays in neuroeducation. Cambridge University Press, Cambridge UK.

Beiras, A. (1998). Estado actual de las Neurociencias. Educación y Neurociencias. (Trabajo de Grado). Universidad de Santiago de Compostela, España,

Blakemore, S. \& Frith, U. (2007). Cómo aprende el cerebro, las claves para la educación (2da. ed.). Madrid: Ariel.

Bruer, J. (1997). Education and the brain: A bridge too far. Rev. Educational, 26(8), 4-16. doi: 10.3102/0013189X026008004

Bruer, J. (2016). Neuroeducación: Un panorama desde el puente. Rev. Propuesta Educativa, 46(2), 14-25

Calzadilla-Pérez, O. (5-7 de noviembre, 2015). La integración de las neurociencias en la malla curricular de la formación inicial de docentes en Cuba. Conferencia pronunciada en el Congreso Internacional Neurociencias, Familia y Educación, Temuco, Chile.

Calzadilla-Pérez, O. (2017). La integración de las neurociencias en la formación inicial de docentes para las carreras de la educación inicial y básica: caso Cuba. Rev. Actualidades Investigativas en Educación, 17(2), 1-27 doi: 10.15517/aie.v17i2.28709

Calzadilla-Pérez, O. Ponce, S., Calzadilla-González, O., Cepena, M., Hidalgo, R., Chávez, N. ... \& Torres, D. (2018). Caracterización de la integración del conocimiento neurocientífico en la formación del profesional de la educación en las especialidades Preescolar, Primaria, Especial y Logopedia de la Licenciatura en Educación. Monografía, proyecto nacional "La formación neurodidáctica del profesional de la Educación Inicial y Básica". Universidad de Holguín, Cuba.

Carvalhoa, D. \& Villas, A. (2018). Neurociências e formação de professores: reflexos na educação e economia. Ensaio: aval. pol. públ. Educ. [online], 26(98), 231-247. doi: 10.1590/S0104-40362018002601120.

Castorina, J.A. (2016). La relación problemática entre Neurociencias y educación. Condiciones y análisis crítico. Rev. Propuesta educativa, 2(46), 26-41

Codina, M. (2014). Neuroeducación en virtudes cordiales. Una propuesta a partir de la Neuroeducación y la ética discursiva cordial. (Tesis doctoral). Valencia: Universidad de Valencia, España. Recuperado de http://roderic.uv.es/handle/10550/35898

Dekker, S., Lee, NC., Howard-Jones, P. \& Jolles, J. (2012). Neuromyths in education: Prevalence and predictors of misconceptions among teachers. Rev. Frontiers in Psychology. 3, 1-8. doi: 10.3389/fpsyg.2012.00429

Falquez, J.F. \& Ocampo, J.C. (2018). Del conocimiento científico al malentendido. Prevalencia de neuromitos en estudiantes ecuatorianos. Rev. Iberoamericana de Educación, 78(1), $87-106$

Fernández, A. (2017). Neurodidáctica e inclusión educativa. Rev. Publicaciones didácticas, 80, 262-266

Ferreira, R.A. (2018). ¿Neurociencia o neuromitos? Avanzando hacia una nueva disciplina. En J. Osorio \& M. Gloël, La didáctica como fundamento de la práctica profesional docente. Tendencias enfoques y avances (pp. 28-46). Editorial Universidad Católica de la Santísima Concepción.

Ferreira, R.A., \& Gómez Álvarez, L. (2019). ¿Por qué la neurociencia debería ser parte de la formación inicial docente? Synergies Chili, 15, 45-56. 
Fuentes, A. \& Risso, A. (2015). Evaluación de conocimientos y actitudes sobre neuromitos en futuros/as maestros/as. Rev. Estudios e investigación en Psicología y Educación, Extr. (6), 1-6. doi: 10.17979/reipe.2015.0.06.530

Geake, J. (2005). Educational neuroscience and neuroscientific education: in search of a middleway. Research Intelligence, 92, 10-13.

Gleichgerrcht, E., Luttges, B.L., Salvarezza, F. \& Campos, A.L. (2015). Educational neuromyths among teachers in Latin America. International Mind, Brain and Education Society, 9(3), 170-178. doi: 10.1111/mbe.12086

Goswami, U. (2004). Neuroscience and education. British Journal of Educational Psychology, $74,1-14$.

Goswami, U. (2006). Neuroscience and education: from research to practice? Nature Reviews Neuroscience, 7(5), 406-413. doi: 10.1038/nrn1907

Hernández, R., Fernández, C., \& Baptista, P. (2014). Metodología de la investigación. México: McGraw-Hill.

Horvath, J. \& Donoghue, G. (2016). A Bridge Too Far - Revisited: Reframing Bruer's neuroeducation Argument for Modern Science of Learning Practitioners. Frontiers in Psychology, 7, 377. doi: 10.3389/fpsyg.2016.00377

Howard-Jones, P. (2010). Introducing Neuroeducational Research. Neuroscience, education and the brain from contexts to practice. Oxon: Routledge.

Howard-Jones, P. (2014). Neuroscience and education: myths and messages. Rev. Nature Reviews Neuroscience, 15(12), 817-824.

Karakus, O., Howard-Jones, P.A. \& Jay T. (2015). Primary and secondary school teachers' knowledge and misconceptions about the brain in Turkey. Procedia-Social and Behavioral Sciences, 174, 1933-1940. doi: 10.1016/j.sbspro.2015.01.858

Lipina, S. (2016). Actualizaciones en neurociencia educacional. Facultad Latinoamericana de Ciencias Sociales. Propuesta Educativa, 2(46), 6-13.

López, J. (2017). Conocimientos neurocientíficos frente a las actitudes de los estudiantes de educación del Instituto Superior Pedagógico Bilingüe de Yarincocha, 2017. Investigación Científica, 2(4), 41-46.

Luque, M. \& García, M. (12-14 de diciembre, 2017). Neurociencias en Ciencias de la Educación. Coordinación y formación docente desde la Universidad. Ponencia presentada I Congreso Virtual Internacional Educacional Innovación y TICs. Recuperado de: https://www.researchgate.net/publication/323366228

Martín, P. (2012). La neurociencia en la formación inicial de educadores: una experiencia innovadora. Participación educativa, 1(1), 92-102.

Melo, T. (1998). Neurociencia + pedagogía $=$ neuropedagogía : repercusiones e implicaciones de los avances de la neurociencia para la práctica educativa (Tesis de Maestría). Universidad Internacional de Andalucía, Sevilla, España. Recuperado de: https://dspace.unia.es/handle/10334/2075

Ministerio de Educación Superior, Cuba [MES] (2016). Formación Pedagógica General para las especialidades Preescolar, Primaria, Especial y Logopedia (Plan de Estudio “E”). Documento inédito. La Habana.

Mora, F. (2013). Neuroeducación: Solo se puede aprender aquello que se ama. Madrid: Alianza Editorial.

Nancekivell, S. E., Shah, P., \& Gelman, S. A. (2020). Maybe they're born with it, or maybe it's experience: Toward a deeper understanding of the learning style myth. Journal of Educational Psychology, 112(2), 221-235. doi: 10.1037/edu0000366

Pei, X., Howard-Jones, PA., Zhang, S., Liu, X., \& Jin, Y. (2014). Teachers' understanding about the brain in East China. Procedia - Social and Behaviour Sciences, 174, 3681-3688. doi: 10.1016/j.sbspro.2015.01.1091 
Rato, J.R., Abreu, A.M. \& Castro-Caldas, A. (2013). Neuromyths in education: what is fact and what is fition for Portuguese teachers? Educational Research, 55(4), 441-453. doi: $10.1080 / 00131881.2013 .844947$

Resende, A. \& Colombo, P. (2018). Diálogos necessários: neurociência, emoções e a formação inicial de profesores. Revista Iberoamericana de Educación, 78(1), 183-204.

Román, F. (2018). Neurociencias y formación de profesores para la educación infantil. RELAdEI. Revista Latinoamérica la de Educación Infantil, 7(1), 88-93.

Román, M. (2013). Conocimientos que tienen las personas docentes sobre neurociencia, e importancia que atribuyen a los aportes de ésta en los procesos de enseñanza aprendizaje (Tesis de maestría). Universidad Estatal a Distancia. Costa Rica. Recuperado de http://repositorio.uned.ac.cr/reuned/handle/120809/953

Tapia, I. (2013). Diseño y aplicación de un módulo de neurociencias para educadoras de párvulos: cambio de actitudes respecto de la disciplina (Tesis posgrado). Universidad de Chile.

Recuperado

de:

http://repositorio.uchile.cl/bitstream/handle/2250/130078/RESUMEN\%20DISENO\%20D E\%20UN\%20MODULO\%20DE\%20NEUROCIENCIAS\%20PARA\%20EDUCADORA S\%20DE\%20PARVULOS\%20CAMBIO\%20DE\%20ACTITUDES\%20RESPECTO $\% 20$ DE\%20LA\%20DISCIPLINA.pdf?sequence $=2$

Tardif, E., Doudin, P.A., \& Meylan, N. (2015). Neuromyths among teachers and student teachers. Rev. Mind, Brain, and Education, 9(1). doi: 10.1111 / mbe.12070

Zabalza, M. (2018). Neurociencias y formación de profesores para la educación infantil. Revista Latinoamérica la de Educación Infantil, 7(1), 78-85.

Zuluaga, J. (2018). Neurociencias y educación. Rev. Latinoamérica la de Educación Infantil, 7(1), 17-33.

Contribución de los autores: a) Concepción y diseño del trabajo; b) Adquisición de datos; c) Análisis e interpretación de datos; d) Redacción del manuscrito; e) revisión crítica del manuscrito.

E.H.J.P. ha contribuido en a,b,c,d,e; O.O.C.P. en c,d,e.

Editora científica responsable: Dra. Cecilia Cracco 